\title{
MAPAS CONCEPTUALES QUE APORTAN AL PROCESO DE ENSEÑANZA - APRENDIZAJE
}

\author{
AUTORES: Luis Fernando Lucio Villacreses ${ }^{1}$ \\ Alfredo Lesvel Castro Landin ${ }^{2}$ \\ Robards Javier Lima Pisco ${ }^{3}$ \\ Mayra Fabiola Regalado Vásquez ${ }^{4}$
}

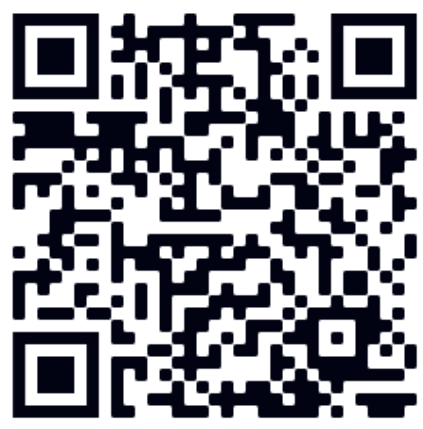

\section{DIRECCIÓN PARA CORRESPONDENCIA: luis.lucio@unesum.edu.ec}

Fecha de recepción: 12/08/2019

Fecha de aceptación: 16/11/2019

\section{RESUMEN}

El presente artículo hace referencia inicial al método sugerido por Joseph D. Novak quien en el año 1972 desarrolló una herramienta a la que denominó mapas conceptuales, y al método creado en el año 2011 por Álvarez Marañón quien desarrolló siete pasos para la creación de un mapa conceptural. En este contexto, basado en la experiencia de 63 estudiantes que cursaron entre Mayo - Septiembre de 2019 el segundo nivel de Ingenieria en Tecnologías de la Información en la Universidad Estatal del Sur de Manabí, se realizó un analisis cualitativo y cuantitativo de los criterios y aspectos utilizados en el desarrollo de un mapa conceptual. Este proceso permitió por una parte, evidenciar que los estudiantes no tenian una guia metodológica definida, por ende se simplificaba el trabajo buscando en la internet mapas ya realizados y los estudiantes que realizaban un mapa conceptual incluian baja calidad y cantidad de información. Por otra parte, se pudo concertar tres procesos vitales a seguir: 1.- Búsqueda y análisis de información de fuentes confiables, que se sustenta con gráficos, fórmulas y ejercicios; 2.- Bosquejo de un diseño original que enlaza la información y/o contenidos seleccionados; 3.- Estructurar, organizar y elaborar el mapa conceptual de manera que contenga una aceptable cantidad y calidad de información, que incluye una exhaustiva revisión ortográfica y fuentes consultadas. Finalmente, el uso de esta técnica influenció en el aprendizaje significativo de las asignaturas de Algebra Lineal y Física, lográndose que el 95,24\% es decir 60 estudiantes sean promovidos al nivel inmediato

PALABRAS CLAVE: gebra; enseñanza; física; significativo

\footnotetext{
${ }^{1}$ Ing. Forestal, Magister en Educación y Desarrollo Social, Docente de la Carrera de Tecnologías de la Información de la Universidad Estatal del Sur de Manabí. Jipijapa, Manabí, Ecuador. luis.lucio@unesum.edu.ecorcid id-00000002-3757-7183

${ }^{2}$ Ing. Agrónomo. Master en Ciencias Agrícolas, Docente en la carrera de Ing. Ambienta. Jipijapa, Manabí, Ecuador. afredolandin55@gmail.com

${ }^{3}$ Economista, Magister en Administración Ambiental. Docente de la Carrera de Tecnologías de la Información de la Universidad Estatal del Sur de Manabí. Jipijapa, Manabí, Ecuador. robardslima@gmail.com ${ }^{4}$ Ing. Comercial. Profesional en libre ejercicio. Manabí. Jipijapa, Manabí, Ecuador. mayfa20@hotmail.com
} 
Luis Fernando Lucio Villacreses, Alfredo Lesvel Castro Landin, Robards Javier Lima Pisco...

\section{ACADEMIC LIVES OF THE STUDENTS OF THE FIRST SEMESTER MATUTINE OF INFORMATION TECHNOLOGIES-UNESUM}

\section{ABSTRACT}

This article makes initial reference to the method suggested by Joseph D. Novak who in 1972 developed a tool he called conceptual maps, and to the method created in 2011 by Álvarez Marañón who developed seven steps to create a map conceptual. In this context, based on the experience of 63 students who completed the second level of Information Technology Engineering at the State University of Southern Manabí between May - September 2019, a qualitative and quantitative analysis of the criteria and aspects was carried out used in the development of a concept map. This process allowed, on the one hand, to demonstrate that the students did not have a defined methodological guide, therefore the work was simplified by searching the internet for already made maps and the students who made a concept map included low quality and quantity of information. On the other hand, three vital processes could be arranged to follow: 1.- Search and analysis of information from reliable sources, which is supported by graphs, formulas and exercises; 2.- Sketch of an original design that links the information and / or selected contents; 3.- Structure, organize and elaborate the concept map so that it contains an acceptable quantity and quality of information, which includes an exhaustive spelling check and consulted sources. Finally, the use of this technique influenced the significant learning of the subjects of Linear and Physical Algebra, achieving that $95.24 \%$, that is, 60 students are promoted to the next higher level

KEYWORDS: algebra learning; physical; significant

\section{INTRODUCCIÓN}

La Constitución de la República del Ecuador (2008), en su artículo 26, estipula que "la educación es un derecho de las personas a lo largo de su vida y un deber ineludible e inexcusable del Estado" y, en su artículo 343, reconoce que el centro de los procesos educativos es el sujeto que aprende; por otra parte, en este mismo artículo se establece que "el sistema nacional de educación integrará una visión intercultural acorde con la diversidad geográfica, cultural y lingüística del país, y el respeto a los derechos de las comunidades, pueblos y nacionalidades".

La Ley Orgánica de Educación Intercultural, en el artículo 2, literal w): "Garantiza el derecho de las personas a una educación de calidad y calidez, pertinente, adecuada, contextualizada, actualizada y articulada en todo el proceso educativo, en sus sistemas, niveles, subniveles o modalidades; y que incluya evaluaciones permanentes. Así mismo, garantiza la concepción del educando como el centro del proceso educativo, con una flexibilidad y propiedad de contenidos, procesos y metodologías que se adapte a sus necesidades y realidades fundamentales. Promueve condiciones adecuadas de respeto, tolerancia y afecto, que generen un clima escolar propicio en el proceso de aprendizaje.".

La Universidad Estatal del Sur de Manabí-UNESUM, empeñada en profesionalizar a todos sus estudiantes, por una parte, crea ambientes de trabajo que cumplan los lineamientos establecidos por el Consejo de Aseguramiento de la Calidad de la Educación Superior-CACES, y por otra toma el modelo pedagógico constructivista y centra sus esfuerzos en lograr que cada docente genere procesos de enseñanza aprendizaje activos, en el cual el estudiante cometerá errores y encontrará soluciones, muchas de estas a través de investigaciones planteadas en el aula o por

34 UNESUM-Ciencias.Publicación cuatrimestral. Vol. 3, Año 2019, No. 2 (Mayo - Agosto) 
necesidad propia del educando, quien se reconoce como autor de su propio aprendizaje y lo relaciona con su entorno inmediato, a través de experiencias, hechos y el entendimiento alcanzado, estructurando finalmente su conocimiento del mundo que le permite crear relaciones racionales y relaciones significativas (Lucio, 2019).

En el contexto expuesto, ha sido y será importante reconocer los aprendizajes de cada estudiante, en este caso, en torno a la elaboración de mapas conceptuales, lo que permitió en el aula de clases consensuar criterios y definir los pasos mínimos que se requieren para realizar un trabajo de investigación y el desarrollo de prácticas de aprendizajesignificativo, que compila información y es presentada por los estudiantes bajo los siguientes criterios: mi mapa conceptual posee aspectos cualitativos que describe la temática investigada, incluye aspectos cuantitativos y gráficos que sustentan la investigación, y finalmente posee un diseño propio de mi autoría, que respeta las fuentes consultadas.

\section{DESARROLLO}

Trabajando sobre la teoría del aprendizaje significativo de Ausubel, Joseph D. Novak desarrolló en 1972 una herramienta de instrucción a la que denominó mapas conceptuales con el objetivo de propiciar un aprendizaje significativo en los alumnos, frente al aprendizaje mecánico o memorístico. Novak conoció a Ausubel y quedó atrapado por el énfasis que éste ponía para explicar el importante papel que juegan los conceptos en el aprendizaje significativo. De hecho, los fundamentos teóricos del mapa conceptual son los mismos que los planteados por Ausubel en su concepción del aprendizaje. En este sentido, el mapa conceptual -según su autor- es una proyección práctica de la teoría de Ausubel. Según Novak "Se consideran una técnica o método de aprendizaje cuya función es ayudar a la comprensión de los conocimientos que el alumno tiene que aprender y a relacionarlos con los que ya posee mediante la representación gráfica y esquemática de un conjunto de relaciones significativas entre conceptos, jerarquizadas según el mayor o menor nivel de abstracción que presentan".

La finalidad del mapa conceptual es representar relaciones significativas entre conceptos; para ello la información está organizada, presentada y representada en niveles de abstracción. Los conceptos más generales o inclusivos se sitúan en la parte superior del esquema, mientras que los más específicos o menos inclusivos, se hallan en la parte inferior. Tal como lo plantea Novak, el mapa conceptual es un instrumento útil para negociar significados. Esto es así porque para aprender el significado de cualquier conocimiento es necesario el intercambio, el diálogo y la discusión. Así, la realización de mapas conceptuales de manera colaborativa promueve la negociación y fomenta la creatividad, al tiempo que pone en valor la siempre útil función social del aprendizaje.

Una reflexión interesante de Novak es que "para que los mapas conceptuales que se presentan en los textos y en las clases resulten beneficiosos, los aprendices deben construir los suyos propios y aprender este modo de organizar los conocimientos". Posteriormente, se descubre su utilidad para ayudar al profesor a organizar el conocimiento y a los alumnos para hallar los conceptos y principios más importantes. (Hernández Tovar \& Mayoral Llorente, n.d.).

\section{Elementos de un mapa conceptual}

De acuerdo con lo planteado por Novak, el mapa conceptual contiene tres elementos fundamentales: 
Luis Fernando Lucio Villacreses, Alfredo Lesvel Castro Landin, Robards Javier Lima Pisco...

Los conceptos: Hacen referencia a acontecimientos y a objetos que se producen en la mente del individuo. No se consideran conceptos los nombres propios, los verbos, los adjetivos y las fechas. Deben estar formados por tres palabras como máximo(Mar Cornelio, Santana Ching et al. 2020), (Mar, Santana et al. 2019).

Palabras-enlace: Son las palabras que se utilizan para vincular los conceptos y representar el tipo de relación que se establece entre ellos. Son las preposiciones, las conjunciones, el adverbio y en general todas las palabras que no sean concepto y que se utilizan para relacionarlos y así construir una "proposición", por ejemplo: para, por, donde, como, entre otras. Las palabras enlace permiten, junto con los conceptos, construir frases u oraciones con significado lógico y hallar la conexión entre conceptos.

Las proposiciones: Forman una unidad semántica que consta de dos o más conceptos unidos por palabras enlace.

Otros elementos: Líneas, flechas de enlace y conexiones cruzadas.

\section{Características de los mapas conceptuales.}

El mapa conceptual es una manera de representar gráficamente la información -o los conceptos- y posee características o condiciones propias, que lo diferencia de otras estrategias o técnicas cognitivas:

Jerarquización: Los conceptos se ordenan de mayor a menor según la importancia o inclusividad. Los de mayor jerarquía se ubican en la parte superior.

Selección: Antes de construir el mapa conceptual hay que seleccionar los conceptos más importantes.

Impacto visual: Relacionado con las características anteriores. En la medida en que el mapa conceptual esté bien elaborado, será más claro, simple y vistoso. Por ese motivo, la distribución espacial de los conceptos es fundamental para la comprensión.

Los mapas conceptuales pueden utilizarse como estrategia de enseñanza, aprendizaje, como recurso para la evaluación, y para la estructuración y organización del currículo. Se ha comentado que Novak creó el mapa conceptual como una fórmula para llevar a la práctica las ideas de Ausubel sobre el aprendizaje significativo. Por este motivo, resulta indispensable trabajar estos cuatro aspectos básicos:

Conexión con las ideas previas de los alumnos.

Inclusión o estructuración jerárquica de los conceptos.

Diferenciación progresiva.

Reconciliación integradora.

\section{Siete pasos para la creación de mapas conceptuales en presentaciones}

Durante la fase de planificación deben identificarse y resumirse los conceptos nucleares de la presentación con el fin de destilar su mensaje principal. Resulta fundamental evitar información irrelevante o detalles secundarios que desbordan a la audiencia impidiéndole captar el mensaje principal. Todo lo que no contribuya a comunicar el mensaje fundamental debe ser eliminado de 
la presentación. Los mapas conceptuales ayudan en este proceso. Veamos cuáles serían los siete pasos para la creación de un mapa conceptual para su uso en presentaciones. (Álvarez Marañón, 2011)

\section{Construcción de una buena idea focal}

Las mejores presentaciones buscan transmitir un único mensaje. En lugar de pretender transferir mucha información, lo que termina diluyendo el mensaje, suele ser mejor intentar transmitir una sola idea. Dejar bien claro desde el principio cuál es la idea nuclear de tu presentación crea un contexto que te ayudará a clasificar jerárquicamente otras ideas secundarias que pueden apoyar la transmisión de tu idea fundamental. Prueba a expresar la idea central de tu presentación en una sola frase. Sugerencia de conceptos relevantes. Una vez que has acotado el dominio de conocimiento y definido el mensaje principal, debes seleccionar los conceptos clave de este dominio necesarios para proporcionar el conocimiento requerido para transmitir la idea focal. $\mathrm{Si}$ partes de un documento escrito, puedes destacar los principales conceptos que contiene.

Lista de conceptos

A continuación, identifica todos los datos, hechos, conceptos, ideas, términos que están asociados de alguna manera con la idea principal y que podrían ayudar a clarificar el mensaje. Puedes crear una lista con estos conceptos, utilizando una sola palabra o una corta frase para cada uno. Se trata como ves de un proceso de torrente de ideas (brainstorming), durante el cual debes anotar absolutamente todo lo que se te ocurra relacionado con la idea focal. No te preocupes en este momento por la redundancia o importancia relativa de los términos y conceptos que van apareciendo. Debe primar la cantidad sobre la calidad. Cuando hayas terminado una primera ronda, habrás compilado una lista inicial de entre 15 y 25 conceptos.

\section{Orden de rango}

Ordena los conceptos poniendo en la parte superior los más relevantes y hacia la inferior los menos importantes. Pueden aparecer nuevos conceptos que no se te ocurrieron en el paso anterior. Puedes eliminar los repetidos o reformular los imprecisos.

\section{Mapa conceptual preliminar}

Comienza a unir los conceptos del paso anterior y a descubrir relaciones entre ellos. Puedes utilizar herramientas informáticas (serán tratadas en una próxima entrada) o post-its de colores. Ahora es momento de refinar la clasificación jerárquica de los conceptos y de eliminar aquellos que se consideran secundarios o irrelevantes para la presentación en concreto (Mar, Santana et al. 2019), (Mar, Vázquez et al. 2015).

\section{Proceso iterativo}

Una vez construido el primer mapa, revísalo. Reordena los conceptos, tanto a nivel jerárquico como de relaciones. Considera si están incluidos todos los conceptos relevantes. En función del tiempo asignado, considera si los conceptos menos relevantes tienen cabida. ¿Se ha colado algún concepto irrelevante o que no guarde relación clara con el resto? ¿Falta algún concepto capital?

\section{Reposicionamiento y refinamiento del mapa conceptual}

Crea el mapa final y realiza los últimos ajustes. El mensaje fundamental de la presentación debería ser fácilmente comprensible: el mapa debería poder comunicarlo con claridad a la 
audiencia, evitando información irrelevante o detalles secundarios que distraen y a menudo oscurecen el mensaje principal.

\section{Materiales y métodos.}

\section{Materiales.}

En el desarrollo del presente estudio se utilizaron diferentes libros de consulta, tanto físicos y digitales de las asignaturas de Física y Algebra Lineal. Esto permitió que los estudiantes del Segundo Nivel, Paralelos A - B de la Carrera de Ingeniería en Tecnologías de la Información de la Universidad Estatal del Sur de Manabí, puedan investigar los contenidos programados en los syllabus correspondientes, y puedan desarrollar los mapas conceptuales que solicitaba el docente por cada unidad temática. Por lo expuesto sumado a la diversidad de materiales utilizados por los estudiantes para la elaboración de cada trabajo, cada uno de los mapas conceptuales desarrollados fue motivo de análisis por cada uno de los estudiantes, lo que generó aportes significativos durante la investigación.

\section{Método.}

Los métodos utilizados en el presente estudio fueron:

Método Inducción-deducción: Este método se aplicó al inicio del periodo académico, para que los estudiantes manejen tres criterios en el desarrollo de mapas conceptuales, estos son: 1.- Analizar la información encontrada: Los conceptos y/o contenidos elegidos deben tener aspectos cualitativos relevantes; 2.- Analizar la información encontrada: Cada concepto y/o contenido debe tener datos cuantitativos, incluyendo fórmulas, y gráficos de ser el caso; 3.- Bosquejar un diseño original: Lo cual promueve autenticidad al trabajo; 4.Estructurar el mapa conceptual, revisar ortografía, incluir fuentes de consultas. Este proceso permitió deducir razonamientos y concluir como habitualmente los estudiantes realizaban mapas conceptuales.

Método Cualitativo, Cuantitativo y Estadístico: Por su parte el Método Cualitativo permitió establecer los pasos mínimos requeridos para elaborar un mapa conceptual que observa los 4 criterios expuestos en el párrafo anterior; en cuanto al uso del Método Cuantitativo se manejó un sondeo con preguntas dirigidas en los Paralelos A - B de la Carrera de Ingeniería en Tecnologías de la Información de la Universidad Estatal del Sur de Manabí, que permitió contabilizar las respuestas obtenidas de los estudiantes; finalmente el uso del Método Estadístico permitió tabular los datos y presentarlos en el apartado de resultados encontrados.

Breve descripción de los participantes: Para el desarrollo del presente trabajo, se contó con la participación de 63 estudiantes, con un promedio de edad de 19 años, que entre el mes de Mayo y Septiembre de 2019 cursaron el Segundo Nivel de la Carrera de Ingeniería en Tecnologías de la Información, perteneciente a la Facultad de Ciencias Técnicas de la Universidad Estatal del Sur de Manabí. En este lapso, los estudiantes involucrados desarrollaron mapas conceptuales para las asignaturas de Física y Algebra Lineal. En el siguiente cuadro se detalla la conformación de género en cada uno de los paralelos involucrados:

Tabla 2. Conformación de género

38 UNESUM-Ciencias.Publicación cuatrimestral. Vol. 3, Año 2019, No. 2 (Mayo - Agosto) 
UNESUM-Ciencias: Revista Científica MultidisciplinariaISSN 2602-8166

MAPAS CONCEPTUALES QUE APORTAN AL PROCESO DE ENSEÑANZA - APRENDIZAJE

\begin{tabular}{|l|l|l|l|}
\hline Paralelo & Masculino & Femenino & Total \\
\hline A & 19 & 11 & 30 \\
\hline B & 25 & 8 & 33 \\
\hline TOTAL & 44 & 19 & 63 \\
\hline$\%$ & $68 \%$ & $32 \%$ & $100 \%$ \\
\hline
\end{tabular}

Elaborado por: Ing. Luis Fernando Lucio Villacreses

Los datos que se muestran a continuación fueron tomados del acta final de calificaciones de los estudiantes que asistieron regularmente a sus estudios:

Tabla 4.Resultados alcanzados al término del Primer Periodo Académico 2019

\begin{tabular}{|c|c|c|c|c|}
\hline \multirow{2}{*}{ Estado actual } & \multicolumn{2}{|c|}{$\begin{array}{lr}\text { Número } & \text { de } \\
\text { estudiantes } & \text { según } \\
\text { paralelo } & \\
\end{array}$} & \multicolumn{2}{|c|}{$\begin{array}{l}\text { Porcentaje } \\
\text { estudiantes } \\
\text { paralelo }\end{array}$} \\
\hline & $\mathbf{A}$ & B & $\mathbf{A}$ & B \\
\hline Retirados & 1 & 0 & $3,33 \%$ & $0,00 \%$ \\
\hline Pérdida de créditos & 2 & 0 & $6,67 \%$ & $0,00 \%$ \\
\hline $\begin{array}{l}\text { Estudiantes que fueron } \\
\text { promovidos a tercer nivel }\end{array}$ & 27 & 33 & $70,00 \%$ & $100,00 \%$ \\
\hline Total & 30 & 33 & $100,00 \%$ & $100,00 \%$ \\
\hline
\end{tabular}

Elaborado por: Ing. Luis Fernando Lucio Villacreses

Criterios y aspectos analizados: El análisis participativo que se realizó con los estudiantes involucrados, permitió establecer acuerdos que regirían la elaboración de mapas conceptuales, cuyos criterios y aspectos relevantes se presentan a continuación:

Tabla 1. Criterios y aspectos considerados para el desarrollo de mapas conceptuales

\begin{tabular}{|c|c|}
\hline Criterios & Aspectos individuales por considerar \\
\hline \multirow[t]{2}{*}{$\begin{array}{l}\text { Búsqueda y análisis de la } \\
\text { información encontrada. }\end{array}$} & $\begin{array}{l}\text { Contar con información de fuentes confiables } \\
\text { como bibliotecas de orden físico y/o digital, a } \\
\text { fin de tomar conceptos y contenidos cuyos } \\
\text { aspectos cualitativos son relevantes e } \\
\text { importantes }\end{array}$ \\
\hline & $\begin{array}{l}\text { Conceptos y argumentos resumidos y } \\
\text { sustentados con gráficos, fórmulas y ejercicios }\end{array}$ \\
\hline $\begin{array}{l}\text { Bosquejar un diseño original: } \\
\text { Poseer un diseño propio del } \\
\text { estudiante, lo cual promueve } \\
\text { autenticidad al trabajo }\end{array}$ & $\begin{array}{l}\text { Establece un diseño original y tiene claro los } \\
\text { enlaces de la información y/o contenidos } \\
\text { seleccionados. }\end{array}$ \\
\hline
\end{tabular}




\begin{tabular}{|l|ll|} 
Estructurar, organizar y elaborar el & $\begin{array}{l}\text { Mapa conceptual con cantidad y calidad de } \\
\text { información aceptable }\end{array}$ \\
${$\cline { 2 - 4 }$} }$ & $\begin{array}{l}\text { Revisa ortografía, incluye fuentes } \\
\text { bibliográficas, }\end{array}$ \\
\hline
\end{tabular}

Elaborado por: Ing. Luis Fernando Lucio Villacreses

\section{Resultados encontrados en la investigación.}

Situación inicial: Con la finalidad de conocer la metodología utilizada para elaborar un mapa conceptual, se realizó una sesión de trabajo que determinó principalmente: que los estudiantes habitualmente no siguen una guía, incidiendo en la presentación de diseños repetitivos y/o símiles, obviándose incluso la revisión ortográfica y la inclusión de la fuente consultada. Todo en su conjunto promueve la elaboración de mapas conceptuales con poca cantidad y calidad de información. El siguiente gráfico muestra todos los resultados encontrados:

\section{Gráfico 1. Aspectos considerados inicialmente en la elaboración de un mapa conceptual.}

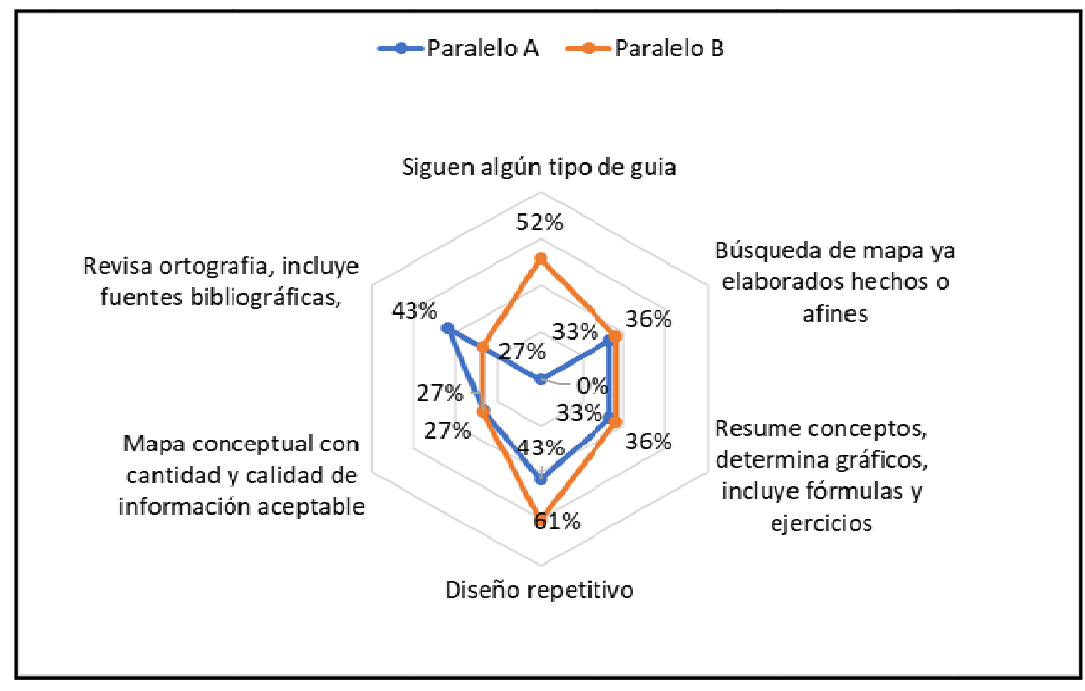

Elaborado por: Ing. Luis Fernando Lucio Villacreses

Situación alcanzada: Al finalizar el primer periodo académico 2019 tras una nueva sesión de trabajo se determinó principalmente: que un alto porcentaje de estudiantes siguió la guía acordada, incidiendo en la presentación de diseños originales que resume conceptos, incluye datos, fórmulas y gráficos, determinando la calidad deseable del mapa conceptual. Esto incluye una alta precaución en la revisión ortográfica y la inclusión de la fuente consultada. Todo en su conjunto aportó significativamente en los procesos de enseñanza - aprendizaje de las asignaturas de Física y Algebra Lineal:

Gráfico 1. Cambios alcanzados en la elaboración de un mapa conceptual. 


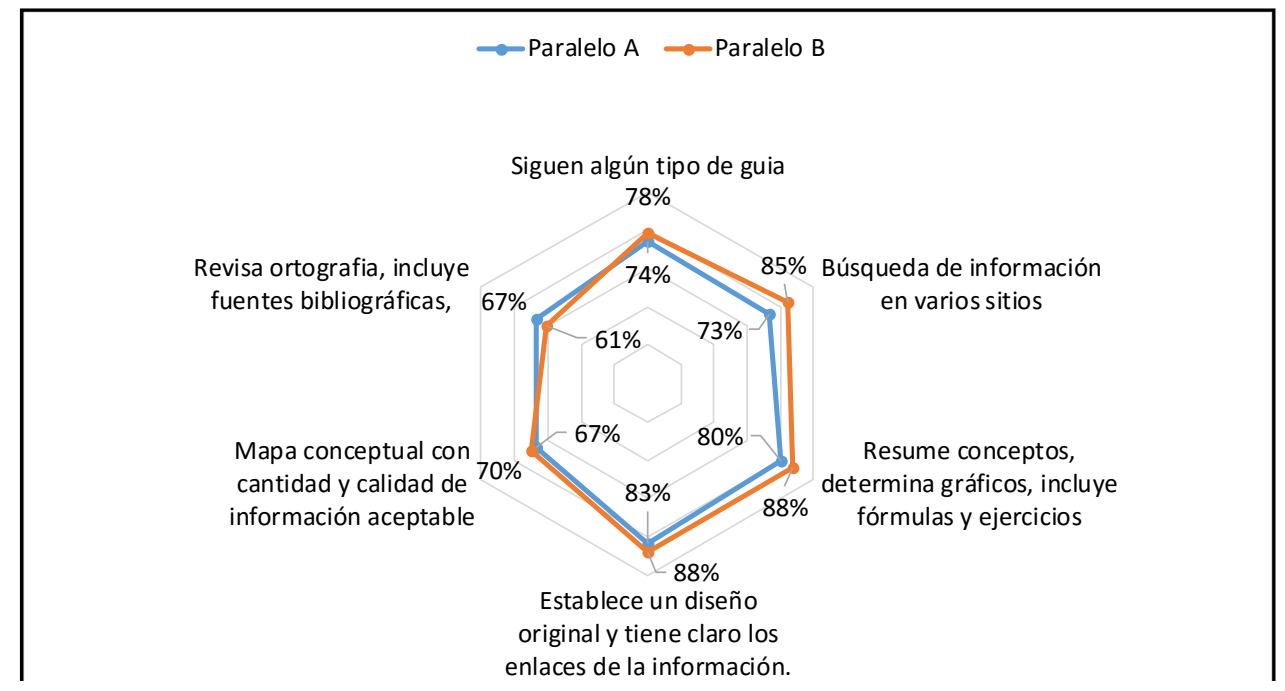

Elaborado por: Ing. Luis Fernando Lucio Villacreses

\section{CONCLUSIONES}

Si bien históricamente han existido procedimientos sugeridos por autores reconocidos como Joseph D. Novak o Álvarez Marañón, estos pasos no son inducidos en el aula de clases por el docente de turno para homologar el uso de una sola herramienta de trabajo, sin embargo aprovechando que el 27\% de los estudiantes Paralelo A y el 52\% de estudiantes del Paralelo B utilizaban algún tipo de guía, se pudo acordar criterios y aspectos a seguir para elaborar mapas conceptuales que permitan un aprendizaje significativo de las asignaturas de Algebra Lineal y Física, evitándose con esto la presentación de mapas copiados de la internet, repetitivos y no relevantes.

Los criterios y aspectos referidos en el presente estudio pueden ser validados y utilizados por toda la colectividad universitaria a fin de conocer las apreciaciones de todos los estudiantes en torno al desarrollo de mapas conceptuales que permiten el aprendizaje significativo de las diferentes asignaturas que se ven en las Carrera profesionalizantes que tiene la Universidad Estatal del Sur de Manabí.

\section{REFERÉNCIAS BIBLIOGRÁFICAS}

Álvarez Marañón, G. (13 de Abril de 2011). El Arte de Presentar. Obtenido de https://www.elartedepresentar.com/2011/04/siete-pasos-para-la-creacion-de-mapasconceptuales-en-presentaciones/

Mar Cornelio, O., I. Santana Ching and J. Gulín González (2020). "Operador por selección para la agregación de información en Mapa Cognitivo Difuso." Revista Cubana de Ciencias Informáticas 14(1): 20-39.

Mar, O., I. Santana and J. Gulín (2019). "Algorithm to determine and eliminate neutral nodes in Cognitive Neutrosophic Map." Neutrosophic Computing and Machine Learning 8: 4-11.

Hernández Tovar, M. J., \& Mayoral Llorente, J. A. (s.f.). http://antia.usal.es/. Obtenido de Universidad de Burgos: 
Luis Fernando Lucio Villacreses; María Fernanda Lucio Hidalgo; Alfredo Lesvel Castro Landin; Robards Javier Lima Pisco; Mayra Fabiola Regalado Vásquez

antia.usal.es/sharedir/tutoriales/Mapasconceptuales/caractersticas_de_los_mapas_co nceptuales.html

Mar, O., I. Santana and J. Gulín (2019). "Algoritmo para determinar y eliminar nodos neutrales en Mapa Cognitivo Neutrosófico." Neutrosophic Computing and Machine Learning 8: 4-11.

Lucio Villacreses, L. F. (2019). Vivencias académicas de los estudiantes de I Semestre Matutino de Tecnología de la Información-Unesum. UNESUM Ciencias.Plan Nacional de Desarrollo. (2017-2021). Sobre los aprendizajes compartidos: Presencia e iniciativas de las instituciones a favor de la educación ambiental . Quito: Educación para el desarrollo sostenible.

Mar, O., M. Y. L. Vázquez and I. S. Ching (2015). "Modelo multicriterio multiexperto utilizando Mapa Cognitivo Difuso para la evaluación de competencias." Ciencias de la Información 46(2): 17-22.

Sampieri. (2018). Metodología de la investigación: las rutas cuantitativa, cualitativa y mixta. Mexico: McGraw Hill Mexico.

42 UNESUM-Ciencias.Publicacióncuatrimestral. Vol. 3. Año 2019.No. 3 (diciembre) 